\title{
On the zeros of functions with finite Dirichlet integral and some related function spaces
}

\author{
By
}

\author{
H. S. SHAPIRO and A. L. SHIELDS *
}

In this paper we consider the class $D$ of functions $f(z)=\sum a_{n} z^{n}$ analytic in the unit circle, with $f(0)=0$, and having finite Dirichlet integral:

$$
\|f\|^{2}=\frac{1}{\pi} \iint_{|z|<1}\left|f^{\prime}(z)\right|^{2} d x d y=\sum_{1}^{\infty} n\left|a_{n}\right|^{2}<\infty .
$$

We wish to know when a set of points $\left\{z_{n}\right\}$ in the unit circle can be the set of zeros of an $f \in D(f \neq 0)$.

In 1952 LENNART CarLeson [5] showed that if

$$
\sum\left(\frac{1}{-\log \left(1-\left|z_{n}\right|\right)}\right)^{1-\varepsilon}<\infty
$$

for some $\varepsilon>0$, then there is an $f \in D$ having these zeros. In the other direction he showed that if $\varepsilon>0$ is given, then there are sets of uniqueness $\left\{z_{n}\right\}$ for which

$$
\sum\left(\frac{1}{-\log \left(1-\left|z_{n}\right|\right)}\right)^{1+\varepsilon}<\infty
$$

(A set $\left\{z_{n}\right\}$ is called a set of uniqueness for the class $D$ if there is no $f \in D$ $(f \neq 0)$ vanishing at all these points.) He noted that an earlier result of LoKKI [9] was incorrect. (The 1955 Ergebnisse tract of WITrICH [13] quotes only the LoKkI result.)

CARLESON also pointed out that if the $\left\{z_{n}\right\}$ all lie on one radius, then the necessary and sufficient condition for the existence of an $f \in D$ with these zeros is: $\sum\left(1-\left|z_{n}\right|\right)<\infty$. Indeed, taking the radius to be the unit interval and letting $B(z)$ be the Blaschke product formed from these points, then $f(z)=(1-z)^{2} B(z)$ has a bounded derivative and so is in $D$.

Our contribution is to show that in (A) one may take $\varepsilon=0$ and that in (B) the expression $\left(-\log \left(1-\left|z_{n}\right|\right)\right)^{-\varepsilon}$ may be replaced by any function tending to zero. Our methods are different from those of CARLEson and make greater use of the fact that $D$ is a Hilbert space. We prove similar theorems

* The first author's contribution to this paper represents research done at the Institute Mathematical Sciences at New York University under the sponsorship of ONR contract N 6 ori-201 (01).

The second author was supported by the Institute of Mathematical Sciences and by the National Science Foundation. 
for certain other Hilbert spaces of analytic functions, where the norm is given by weighting the Taylor coefficients.

We wish to acknowledge helpful correspondence with Professor CarLeson; in particular, Lemma 4 and Theorem 3 were known to him. We benefited also from discussion with D. J. NewMaN.

I. Hilbert space background. We shall need some elementary facts about Hilbert space. Much of this material is presented in the first chapter of ACHIEZER [1].

Let $H$ be a Hilbert space, let $x_{0}, x_{1}, x_{2}, \ldots$ be a linearly independent set of elements of $H$, and let $c_{0}, c_{1}, c_{2}, \ldots$ be complex numbers such that there exists an element $y \in H$ satisfying

$$
\left(y, x_{n}\right)=\dot{c}_{n} \quad(n \geqq 0) .
$$

1. The element $y_{n}$ of minimal norm satisfying

$$
\left(y_{n}, x_{i}\right)=c_{i} \quad(i=0,1,2, \ldots, n)
$$

is a linear combination of $x_{0}, x_{1}, \ldots, x_{n}$.

2. If $y$ is the element of minimal norm satisfying (*), then $y_{n} \rightarrow y$ in norm.

3. Let $c_{0}=1, c_{i}=0(i \geqq 1)$, let $d_{n}$ be the distance from $x_{0}$ to the subspace spanned by $x_{1}, \ldots, x_{n}$ and let $d$ be the distance from $x_{0}$ to the subspace spanned by $x_{1}, x_{2}, \ldots$. Then

$$
\left\|y_{n s}\right\|=\frac{1}{d_{n}} \text { and }\|y\|=\frac{1}{d} .
$$

4. Let $G\left(x_{1}, \ldots, x_{n}\right)$ denote the Gram determinant

Then

$$
G\left(x_{1}, \ldots, x_{n}\right)=\operatorname{det}\left\|\left(x_{i}, x_{j}\right)\right\| \quad(i, j=1, \ldots, n) .
$$

$$
d_{n}^{2}=\frac{G\left(x_{0}, x_{1}, \ldots, x_{n}\right)}{G\left(x_{1}, \ldots, x_{n}\right)} .
$$

The denominator is positive since the $x_{i}$ are linearly independent.

II. Functions with finite Dirichlet integral. Let $D$ denoto the Hilbert space of functions $f(z)=\sum a_{n} z^{n}(f(0)=0)$ analytic in the unit circle with finite Dirichlet integral. The inner product is given by

where $g(z)=\sum b_{n} z^{n}$

$$
(f, g)=\sum_{1}^{\infty} n a_{n} \bar{b}_{n}
$$

The function

$$
K_{\zeta}(z)=\log \frac{1}{1-\bar{\zeta} z}=\sum_{1}^{\infty} \frac{1}{n}(\bar{\zeta} z)^{n}
$$

is the "reproducing kernel" for the space $D$, that is,

$$
f(\zeta)=\left(f, K_{\zeta}\right)
$$


for all $f \in D$ and all $|\zeta|<1$. (See AronszajN [2] and [3] for the general theory of kernel functions.) Also,

$$
\left\|K_{\zeta}\right\|^{2}=\left(K_{\zeta}, K_{\zeta}\right)=K_{\zeta}(\zeta)=-\log \left(1-|\zeta|^{2}\right)
$$

by (1) and (2). Thus:

$$
|f(\zeta)|=\left|\left(f, K_{\zeta}\right)\right| \leqq\|f\| \cdot\left\|K_{\zeta}\right\|=\|f\|\left(\log \frac{1}{1-|\zeta|^{2}}\right)^{\frac{1}{2}}
$$

for all $f \in D$, with equality only for $f=c K_{\zeta}$.

We shall need the fact that any finite set of kernel functions corresponding to distinct points is linearly independent. Indeed, let $z_{1}, \ldots, z_{n}$ be distinct points in the unit circle (all different from zero) and let $K_{j}$ be the corresponding kernel functions. If one had a dependence relation, say $K_{1}=c_{2} K_{2}+\cdots$ $+c_{n} \dot{K}_{n}$, then each $f \in D$ vanishing at $z_{2}, \ldots, z_{n}$ would vanish at $z_{1}$ also. But this is false since $D$ contains all polynomials vanishing at the origin.

Now let $z_{1}, z_{2}, \ldots$ be distinct points inside the unit circle, all different from zero. If there is a function $f \in D$ vanishing at these points but not vanishing identically, then there is an $f \in D$ vanishing at these points with $f^{\prime}(0)=1$. In other words, putting $K_{n}=K_{z_{n}}$ we have

$$
(f, z)=1, \quad\left(f, K_{n}\right)=0 \quad(n=1,2, \ldots) .
$$

This is equivalent to saying that the function $z$ is at a positive distance $d$ from the subspace spanned by $K_{1}, K_{2}, \ldots$ :

$$
d \geqq \frac{1}{\|f\|}
$$

The square of the distance from $z$ to the subspace spanned by $K_{1}, \ldots, K_{n}$ is given by the quotient of two Gram determinants (see Section I.4):

$$
d_{n}^{2}=\frac{\left|\begin{array}{cccc}
1 & z_{1} & \ldots & z_{n} \\
\bar{z}_{1} & -\log \left(1-\left|z_{1}\right|^{2}\right) & \ldots & -\log \left(1-\bar{z}_{1}, z\right) \\
\vdots & & & \\
\bar{z}_{n} & -\log \left(1-\bar{z}_{n} z_{1}\right) & \ldots & -\log \left(1-\left|z_{n}\right|^{2}\right)
\end{array}\right|}{G\left(K_{1}, \ldots, K_{n}\right)} .
$$

Multiply the first row in the numerator by $\bar{z}_{i}$ and subtract it from the $i$-th row. When this has been done for each $i$ the numerator becomes:

$$
\left\{\begin{array}{cccc}
\left|\begin{array}{cccc}
1 & z_{1} & \ldots & z_{n} \\
0 & h\left(z_{1} \bar{z}_{1}\right) & \ldots & h\left(z_{n} \bar{z}_{1}\right) \\
\vdots & & & \\
0 & h\left(z_{1} \bar{z}_{n}\right) & \ldots & h\left(z_{n} \bar{z}_{n}\right)
\end{array}\right|=\operatorname{det}\left\|h\left(z_{i} \bar{z}_{j}\right)\right\| \\
\quad=\operatorname{det}\left\|-\log \left(1-z_{i} \bar{z}_{j}\right)\left[1+\frac{z_{i} \bar{z}_{j}}{\log \left(1-z_{i} \bar{z}_{j}\right)}\right]\right\|
\end{array}\right.
$$

where $h(w)=-w-\log (1-w)$. 
Now, det $\left\|-\log \left(1-z_{i} \bar{z}_{j}\right)\right\|$ is a Gram determinant (the denominator of $(5)$ ) and hence is positive definite. We wish to show that $\left\|1+z_{i} \bar{z}_{j} / \log \left(1-z_{i} \bar{z}_{j}\right)\right\|$ is positive semi-definite. For this we need the following two lemmas, the first of which is given in HARDY [7], Chapter IV, Theorem 2.2

LEMMA 1. Let $p(x)=1+a_{1} x+a_{2} x^{2}+\cdots$ where $a_{n}>0, \quad a_{n+1} a_{n-1} \geqq a_{n}^{2}$ $(n=1,2, \ldots)$. Then $1-1 / p(x)$ has non-negative Taylor coefficients.

The function $p(x)=-[\log (1-x)] / x$ satisfies the conditions of the lemma, and so $1+x / \log (1-x)$ has non-negative Taylor coefficients.

LEMMA 2. If $g(w)=\sum a_{n} w^{n}(|w|<1)$ has non-negative Taylor coefficients, and if $z_{1}, z_{2}, \ldots, z_{n}\left(\left|z_{i}\right|<1\right)$ are complex numbers, then the matrix $A=\left\|g\left(z_{i} \bar{z}_{j}\right)\right\|$ is positive semi-definite.

Proof. $A=\sum a_{n}\left\|\left(z_{i} \bar{z}_{j}\right)^{n}\right\|$. The matrix $\left\|\left(z_{i} \bar{z}_{j}\right)^{n}\right\|$ corresponds to a positive semi-definite quadratic form, since for any choice of complex numbers $w_{1}, \ldots, w_{n}$ we have

$$
\sum_{i, j}\left(z_{i} \bar{z}_{j}\right)^{n} w_{i} w_{j}=\left|\sum_{i} z_{i}^{n} w_{i}\right|^{2} \geqq 0
$$

Multiplying by $a_{n}$ and summing on $n$ we see that $A$ is semi-definite, which completes the proof of the lemma.

We are now in a position to use the following result of OPPENHEIm [10] (see also ScHur [12]).

LEMMA 3. Let $\left\|a_{i j}\right\|,\left\|b_{i j}\right\|$ be positive semi-definite square matrices. Then

$$
\operatorname{det}\left\|a_{i j} b_{i j}\right\| \geqq\left(\operatorname{det}\left\|a_{i j}\right\|\right)\left(\prod b_{i i}\right) \text {. }
$$

Applied to (6) this yields

$$
d_{n}^{2} \geqq \prod_{1}^{n}\left(1+\frac{\left|z_{i}\right|^{2}}{\log \left(1-\left|z_{i}\right|^{2}\right)}\right) .
$$

Thus we have proven the following theorem.

THEOREM 1. If $\left\{z_{n}\right\}$ is a sequence of points in the unit circle for which

$$
\sum \frac{1}{-\log \left(1-\left|z_{n}\right|^{2}\right)}<\infty
$$

then there is an $f \in D$ vanishing at these points, with $f^{\prime}(0)=1$, and

$$
\|f\|^{2} \leqq \prod_{1}^{\infty}\left(1+\frac{\left|z_{n}\right|^{2}}{\log \left(1-\left|z_{n}\right|^{2}\right)}\right)^{-1} .
$$

(Strictly speaking our proof was only for the case when the $\left\{z_{n}\right\}$ are all distinct and different from zero. In view of (9), however, the case of repeated $z_{n}$ can be treated by a passage to the limit. Finally, if some of the $z_{n}$ are zero we first find an $f$ vanishing at the remaining points and then multiply it by a power of $z$.)

Actually a slightly stronger result is true. Under the conditions of the theorem there is an $f \in D$ having these zeros and no others in the unit circle. 
This follows from the fact (see Lokk $[9]$, p. 27) that if $f \in D$ then

$$
\left\|f(z) \frac{z-a}{1-a z}\right\|>\|f\| \text {. }
$$

Thus the function of minimal norm vanishing at the points $\left\{z_{n}\right\}$, with $f^{\prime}(0)=1$, has no other zeros.

(Inequality (10) is obvious when $a=0$, using the expression for the norm in terms of the Taylor coefficients. The general case follows by a conformal transformation, since the norm is invariant under the substitution

$$
z \rightarrow(z-a) /(1-\bar{a} z)
$$

THEOREM 2. Let $\left\{z_{n}\right\}$ be a sequence of distinct points in the unit circle, all different from zero, and let there be an $f \in D(f \neq 0)$ vanishing at these points. Then the functions

$$
f_{n}(z)=\frac{\left|\begin{array}{cccc}
z & z_{1} & \ldots & z_{n} \\
K_{1}(z) & \left(K_{1}, K_{1}\right) & \ldots & \left(K_{1}, K_{n}\right) \\
\vdots & & & \\
K_{n}(z) & \left(K_{n}, K_{1}\right) & \ldots & \left(K_{n}, K_{n}\right)
\end{array}\right|}{d_{n}^{2} G\left(K_{1}, \ldots, K_{n}\right)}
$$

(see (5)) are in $D$ and have the following properties:

(i) $f_{n}\left(z_{i}\right)=\left(f_{n}, K_{i}\right)=0 \quad(1 \leqq i \leqq n)$;

.(ii) $f_{n}^{\prime}(0)=\left(f_{n}, z\right)=1$;

(iii) $f_{n}$ has minimal norm among all functions in $D$ satisfying (i) and (ii), and $\left\|f_{n}\right\|=1 / d_{n}$;

(iv) $f_{n}$ converges in norm to the unique $f \in D$ of minimal norm, vanishing on the set $\left\{z_{n}\right\}$, with $f^{\prime}(0)=1$.

Properties (i) and (ii) are obvious, (iii) follows from Sections I.1 and I.3, and (iv) follows from I.2.

In the case of the Hilbert space $H_{2}$ the partial products of the Blaschke product are the solutions to a similar extremal problem: among all functions of norm one vanishing at $z_{1}, \ldots, z_{n}$, find the function that maximizes $|f(0)|$.

In the converse direction to Theorem 1 we have the following result.

THEOREM 3. Let $h(t)$ be any continuous function with $h(0)=0, h(t)>0$ $(t>0)$. Then there exists a set of uniqueness $\left\{z_{n}\right\}$ satisfying the condition

$$
\sum \frac{1}{-\log \left(1-\left|z_{n}\right|\right)} h\left(1-\left|z_{n}\right|\right)<\infty \text {. }
$$

The proof of this result is based on the following two lemmas.

LemMA 4. Let $r$ be a positive number less than 1 , and let $z_{1}, z_{2}, \ldots, z_{n}$ be equally spaced points on the circle $|z|=r$. If $f\left(z_{i}\right)=0(i \leqq n)$ and $f^{\prime}(0)=1$ then

$$
\|f\|^{2} \geqq \frac{n}{-\log \left(1-r^{2 n}\right)} .
$$


Proor. Take $z_{1}=r$ (the norm is invariant under rotations). Let $h(z)$ be defined by

Then $(f, h)=0$ and so

$$
h=\frac{1}{n}\left(\frac{K_{1}}{z_{1}}+\cdots+\frac{K_{n}}{z_{n}}\right) .
$$

Also,

$$
1=(f, z)=(f, z-h) \leqq\|f\|\|z-h\| .
$$

and therefore

$$
h(z)-z=\sum_{m=1}^{\infty} \frac{r^{m+1}}{n \cdot m+1} 2^{n m+1}
$$

$$
\|h-z\|=r^{2} \sum_{m=1}^{\infty} \frac{r^{2 n m}}{n m+1}<\frac{1}{n} \log \frac{1}{1-r^{2 n}}
$$

from which the result follows.

LEMMA 5. Let $n_{k} \rightarrow \infty$ be positive integers, let $\delta_{k} \rightarrow 0$ be positive numbers such that $n_{k} \delta_{k} \rightarrow 0$, and let $\varphi(k)$ be determined by

$$
\delta_{k}=\exp \left(-n_{k} \varphi(k)\right) \text {. }
$$

Then, $\varphi(k) \rightarrow 0$ if and only if

$$
\frac{1}{n_{k}} \log \left[1-\left(1-\delta_{k}\right)^{n_{k}}\right] \rightarrow 0 .
$$

This follows from the inequalities

$$
1-n_{k} \delta_{k} \leqq\left(1-\delta_{k}\right)^{n_{k}} \leqq 1-n_{k} \delta_{k}+\frac{1}{2} n_{k}\left(n_{k}-1\right) \delta_{k}^{2} .
$$

ProOF OF THEOREM 3. We shall construct two sequences $\left\{r_{k}\right\}$ and $\left\{n_{k}\right\}$ where $n_{k} \rightarrow \infty$ are positive integers, and $0<r_{k}<1, r_{k} \rightarrow 1$. On the circle of radius $r_{k}$ we take $n_{k}$ points equally spaced. The union of these finite point sets will be the required set of uniqueness $\left\{z_{k}\right\}$.

Let $1-r_{k}=\delta_{k}$ and let $\delta_{k}=\exp \left(-n_{k} \varphi(k)\right)$, where $\varphi(k)$ and $n_{k}$ are to be determined so as to satisfy the following three conditions:

(i) $\varphi(k) \rightarrow 0$;

(ii) $n_{k} \delta_{k} \rightarrow 0$;

(iii) $\sum h\left(\delta_{k}\right) /(\varphi(k))<\infty$.

This will be sufficient to prove the theorem, since (i) and (ii) together with Lemmas 4 and 5 guarantee that $\left\{z_{n}\right\}$ will be a set of uniqueness, while condition (iii) is equivalent to (11).

Let $g(y)=h\left(e^{-y}\right)$. Then $g \rightarrow 0$ as $y \rightarrow \infty$. Let $y_{1}<y_{2}<\cdots$ be integers such that $y_{k} \geqq k$ and $g(y) \leqq\left(\frac{1}{2}\right)^{k}\left(y \geqq y_{k}\right)$. Let $n_{k}=k y_{k}$ and let $\varphi(k)=1 / k$. Then $n_{k} \delta_{k} \leqq y_{k}^{2} \exp \left(-y_{k}\right) \rightarrow 0$ and so (ii) is satisfied. Also

$$
\sum h\left(\delta_{k}\right) / \varphi(k)=\sum g\left(n_{k} \varphi(k)\right) / \varphi(k) \leqq \sum k / 2^{k}<\infty,
$$

and so (iii) is satisfied. This completes the proof of the theorem. 
III. Generalizations. In this section we define a class of Hilbert spaces $D_{\varphi}$, depending on a function $\varphi$. Theorem 1 extends to these spaces. Strictly speaking the space $D$ is not of the type studied here because of the normalization $f(0)=0$. One could, however, consider the space of functions $f(z) / z$ $(f \in D)$, and take for $\varphi$ the function $[-\log (1-z)] / z$.

Let $\varphi(z)=\sum c_{n} z^{n}$ be analytic for $|z|<1$, with $c_{0}=1, c_{n}>0$ and

$$
c_{n}^{2} \leqq c_{n-1} c_{n+1} \quad(n=1,2, \ldots) .
$$

(For example, $c_{n}=1 / n^{\alpha}, \alpha \geqq 0$ ) Let $D_{\varphi}$ be the Hilbert space of functions $f(z)=\sum a_{n} z^{n}$ analytic in $|z|<1$ with norm:

$$
\|f\|^{2}=\sum \frac{1}{c_{n}}\left|a_{n}\right|^{2}<\infty .
$$

If $g=\sum b_{n} z^{n} \in D_{\varphi}$ the inner product $(f, g)$ is given by:

$$
(f, g)=\sum \frac{1}{c_{n}} a_{n} \bar{b}_{n} .
$$

The reproducing kernel is the function

$$
K_{\zeta}(z)=\varphi(\bar{\zeta} z)
$$

and we have $f(\zeta)=\left(f, K_{\zeta}\right)$ for all $f \in D_{\varphi},|\zeta|<1$. In particular, $\left\|K_{\zeta}\right\|^{2}=$ $\left(K_{\zeta}, K_{\zeta}\right)=K_{\zeta}(\zeta)=\varphi\left(|\zeta|^{2}\right)$. Thus

$$
|f(\zeta)|=\left|\left(f, K_{\zeta}\right)\right| \leqq\|f\|\left[\varphi\left(|\zeta|^{2}\right)\right]^{\frac{1}{2}}
$$

for all $t \in D_{\varphi}$.

Lemma 6. If (12) holds and if $\varphi(z)$ is analytic for $|z|<1$ then $1=c_{0} \geqq c_{1} \geqq$ $c_{2} \geqq \cdots$ with equality for one index implying equality for all larger indices. Thus $\varphi(r) \leqq 1 /(1-r)$.

Proof. Let the index $n$ be fixed. Then

$$
c_{n+k} \geqq\left(\frac{c_{n}}{c_{n-1}}\right)^{k} c_{n} \quad(k=0,1,2, \ldots) .
$$

This follows by induction. Indeed, if (14) holds for a particular value of $k$ and if we multiply both sides by $c_{n+k}$ and apply (12) we obtain

$$
c_{n+k+1} \geqq\left(\frac{c_{n}}{c_{n-1}}\right)^{k}\left(\frac{c_{n+k}}{c_{n+k-1}}\right) c_{n} .
$$

But (12) says that $c_{m} / c_{m-1}$ is non-decreasing, and therefore $c_{n+k} / c_{n+k-1} \geqq c_{n} / c_{n-1}$. Thus (15) becomes (14) with $k+1$ instead of $k$.

If now $c_{n}<c_{n-1}$, then from (14) the radius of convergence of $\sum c_{n} z_{n}$ would be less than one, contrary to assumption. Therefore $c_{0} \geqq c_{1} \geqq \cdots$. If equality holds for some $n, c_{n}=c_{n+1}$, then from (12) we have $c_{n+1} \leqq c_{n+2}$ and therefore $c_{n+1}=c_{n+2}$, and so forth.

Lemma 7. If $t \in D_{\varphi}$ then

$$
\|f\|^{2} \leqq\|z f\|^{2} \leqq \frac{1}{c_{1}}\|f\|^{2}
$$


Proof. Let $f(z)=\sum a_{n} z^{n}$. Then

$$
\|z f\|^{2}=\sum \frac{\left|a_{n}\right|^{2}}{c_{n+1}}=\sum \frac{\left|a_{n}\right|^{2}}{c_{n}} \frac{c_{n}}{c_{n+1}} .
$$

From Lemma 6 we have $c_{n} / c_{n+1} \geqq 1$, while from (12) we have $c_{n} / c_{n+1} \leqq c_{n-1} / c_{n} \leqq$ $\cdots \leqq c_{0} / c_{1}=1 / c_{1}$, and the result follows.

THEOREM 1'. If $\left\{z_{n}\right\}$ is any sequence of points in the unit circle for which

$$
\sum \frac{1}{\varphi\left(\left|z_{n}\right|^{2}\right)}<\infty
$$

then there is an $f \in D_{\varphi}(f \neq 0)$ vanishing at all these points.

The proof is almost exactly the same as the proof of Theorem 1. Assume that the $\left\{z_{n}\right\}$ are all distinct (the general case is a limiting case of this) and all different from zero (multiplication by $z$ is permissable by Lemma 7). If there is an $f \in D_{\varphi}$ vanishing at these points then we may assume $f(0)=1$. In other words, $(f, 1)=1,\left(f, K_{z_{n}}\right)=0(n=1,2, \ldots)$. The proof now proceeds as in Theorem 1.

As an example, consider the functions

$$
\varphi_{\alpha}(z)=\frac{1}{(1-\bar{\xi} z)^{1-\alpha}} \quad(0 \leqq \alpha<1) .
$$

The corresponding Hilbert spaces will be denoted $D_{\alpha}$. For these spaces Theorem $1^{\prime}$ is not new. In fact, CARLEson showed in this thesis ([4], Chap. 2, Theorem 2.2) that if $\sum\left(1-\left|z_{n}\right|\right)^{1-\alpha}<\infty$ then $B(z) \in D_{\alpha}$, where $B$ is the Blaschke product formed from these zeros. In addition, if $f$ is any other function in $D_{\alpha}$ having these zeros, then $f / B \in D_{\alpha}$. (An infinite Blaschke product $B(z)$ is never in the space $D$, however it follows from (10) that if $f=B g \in D$ then $g \in D$ and $\|g\|<\|f\|$.)

The analogue of Theorem 3 holds for the spaces $D_{\alpha}$ with a similar proof. We do not know if such a theorem is true for all the spaces $D_{\varphi}$ (the trouble comes in extending Lemma 4).

IV: We turn now to functions analytic in the unit circle that satisfy

$$
|f(z)| \leqq \frac{c}{(1-|z|)^{k}} \quad(|z|<1)
$$

for some constants $c, k>0$. This includes, for example, the "Bergmann space" $A_{2}$ of Taylor series $f(z)=\sum a_{n} z^{n}$ satisfying

$$
\|f\|^{2}=\sum_{0}^{\infty} \frac{\left|a_{n}\right|^{2}}{n+1}=\frac{1}{\pi} \iint_{|z|<1}|f(z)|^{2} d x d y<\infty .
$$

The kernel function for $A_{2}$ is $1 /(1-\bar{\zeta} z)^{2}$, and so one has

$$
|f(z)| \leqq \frac{\|f\|}{1-|z|^{2}}\left(f \in A_{2}\right) \text {. }
$$

The class of functions satisfying (16) is identical with the class of Taylor series satisfying $\sum\left|a_{n}\right|^{2} / n^{k}<\infty$ for some constant $k$. 
We present three results about the zeros of functions satisfying (16). The first is a special case of a result of HAYMAN [8] (Theorem IV), and is valid for a wider class of functions; the proof given here was suggested by L. RuBEL.

THEOREM 4. Let $f$ be analytic in the unit circle, and let $0<z_{1} \leqq z_{2} \leqq \cdots$ be those zeros of $f$ that lie on the unit interval. If

$$
|f(z)| \leqq c \exp \frac{1}{(1-|z|)^{\alpha}}
$$

for some $\alpha<\frac{1}{2}$ and some $c>0$, then $\sum\left(1-z_{n}\right)<\infty$.

Proof. Let $C$ denote the disc $\left|z-\frac{1}{2}\right|<\frac{1}{2}$, and let $d_{r}$ denote the circles $\left|z-\frac{1}{2}\right|=r\left(0<r \leqq \frac{1}{2}\right)$. We shall show that

$$
\int_{d_{r}} \log ^{+}|f(z)||d z| \leqq \text { const } \quad\left(0<r \leqq \frac{1}{2}\right) .
$$

Indeed, let $z$ be a point on $d_{r}$ and let $t=t(z)$ be the arc length along $d_{r}$ from the point $z$ to the point $r$. It is a simple fact that the ratio $t^{2} /(1-|z|)$ remains bounded independent of $r\left(0<r \leqq \frac{1}{2}\right)$. Hence if we regard $f$ as a function of $t$ defined on $d_{r}$ we have $\log |f(t)|=O\left(t^{-2} \alpha\right)$, from which (18) follows.

Thus $f$ is a function of bounded characteristic see (Privaloff [11], Chapter 2) relative to the disc $C$, and the result follows from this.

This is far from being the best possible result. For example, in (17) one can take $\alpha<1$ (the proof is similar, but the disc $C$ must be replaced by a region making a lower order of contact with the unit circle). D. J. NEwMAN has conjectured that the conclusion of Theorem 4 is still valid when (17) is replaced by

$$
\int_{0}^{1} \log M_{f}(r) d r<\infty .
$$

THEOREM 5. Let $f$ be analytic in the unit circle and satisfy (16). Let $n(r)$ denote the number of zeros of $f$ in the circle $|z|<r$. Then

$$
n(r)=O\left(\frac{1}{1-r} \log \frac{1}{1-r}\right) .
$$

Proof. Assume without loss of generality that $f(0)=1$. Then from Jensen's formula we have:

$$
\int_{0}^{r} n(x) d x \leqq \int_{0}^{r} \frac{n(x)}{x} d x=\frac{1}{2 \pi i} \int_{0}^{2 \pi} \log \left|f\left(r e^{i \theta}\right)\right| d \vartheta \leqq c \log \frac{1}{1-r} .
$$

Therefore

and the result follows.

$$
n\left(r^{2}\right)\left(r-r^{2}\right) \leqq \int_{r^{2}}^{r} n(x) d x \leqq c \log \frac{1}{1-r},
$$

The next theorem is a modification of an example suggested by D.J. Newman; a similar example was suggested by G. Piranian. 
THEOREM 6. Let $\varepsilon>0$ be given. Then there is a function $f(z)=\sum a_{n} z^{n}$ and a sequence $r_{k} \rightarrow 1$ such that

and

$$
\sum\left|a_{n}\right|^{2} / n^{\varepsilon}<\infty
$$

$$
n\left(r_{k}\right) \geqq \text { const. } \frac{1}{1-r_{k}} \log \frac{1}{1-r_{k}} .
$$

Proof. We shall construct a power series

$$
f(z)=\sum a_{k} z^{n_{i}} \quad|z|<1
$$

with positive coefficients, and a sequence $r_{k} \rightarrow 1$ such that

$$
\begin{gathered}
n_{k} \geqq c \frac{1}{1-r_{k}} \log \frac{1}{1-r_{k}}, \\
a_{k} r_{k}^{n_{k}}>\sum_{j \neq k} a_{j} r_{k}^{n_{k}}, \quad(k=1,2, \ldots), \\
\sum \frac{\left|a_{k}\right|^{2}}{n_{k}^{\varepsilon}}<\infty .
\end{gathered}
$$

Using Rouche's theorem it follows from (i) and (ii) that (19) holds.

Let $\alpha$ be given, $\frac{1}{2^{\varepsilon}}<\alpha<1$. Let $g(k)$ be an increasing sequence of positive integers, which will be determined later. Define the sequence $r_{k}$ by

Then

$$
r_{\hat{k}}^{\phi(k)}=\alpha \text {. }
$$

Let

$$
\frac{1}{1-r_{k}} \sim \text { const. } 4^{g(k)} .
$$

$$
n_{k}=g(k) 4^{g(k)} \text { and } \quad a_{k}=2^{e g(k)} .
$$

Then (i) is satisfied, and (iii) will also hold provided that

$$
\sum \frac{1}{[g(k)]^{8}}<\infty .
$$

To satisfy (ii) it will be sufficient to have:

Now

$$
a_{k} r_{k}^{n_{k}} \geqq \sum_{j<k} a_{j}+\sum_{j>k} a_{j} r_{k}^{n_{j}}
$$

and

$$
a_{k} n_{k}^{n_{k}}=\left(2^{\varepsilon} \alpha\right)^{g(k)},
$$

Let

$$
\sum_{j<k} a_{j}<(k-1) 2^{e g^{(k-1)}} .
$$

Then

$$
\beta_{k}=\alpha^{4 g(k+1)-g(k)} .
$$

$$
\sum_{i>k} a_{i} n_{k}^{n_{j}}<\sum_{i<k}\left(2^{\varepsilon} \beta_{k}\right)^{g(i)} .
$$


In view of all this, (ii) will be satisfied if we choose $g(k)$ so that

$$
2^{\varepsilon} \beta_{k}<\frac{1}{2} \quad \text { and } \quad\left(2^{\varepsilon} \alpha\right)^{g(k)}>(k-1) 2^{\varepsilon g(k-1)}+1
$$

for all $k$. It is clearly possible to choose $g(k)$ to satisfy both (20) and (21). QED.

V. By setting up isometries between the Hilbert spaces studied in this paper and some other ones, it is possible to relate the study of sets of uniqueness to other closure problems. We give some examples of this procedure.

1. Let $D^{\prime}$ denote the Hilbert space of Taylor series $\sum a_{n} z^{n}$ with $\left\|z^{n}\right\|^{2}=n+\frac{1}{2}$, $n=0,1, \ldots\left(D^{\prime}\right.$ is a slight modification of $D$, and has clearly the same sets of uniqueness). Let us set up an isometry between $D^{\prime}$ and $L_{2}(-1,1)$ by the correspondence $z^{n} / \sqrt{n+\frac{1}{2}} \leftrightarrow \sqrt{n+\frac{1}{2}} P_{n}(t)$, where $P_{n}(t)$ is the $n^{\text {th }}$ Legendre polynomial (throughout this section we conform to the notations and normalizations of Tricomi, Vorlesungen über Orthogonalreihen). Since the corresponding elements are orthonormal bases in the respective spaces, this correspondence does indeed induce an isometry. The kernel function of $D^{\prime}$ is mapped onto

$$
\sum_{n=0}^{\infty} P_{n}(t) \bar{\zeta}^{n}=\frac{1}{1-2 t \bar{\zeta}+\bar{\zeta}^{2}} .
$$

Hence, since sets of uniqueness are preserved under complex conjugation, we can state:

A sequence of points $z_{n}$ is a set of uniqueness for the space $D$ if and only if the functions $1 / \sqrt{t-\lambda_{n}}$ are complete in $L_{2}(-1,1)$, where $\lambda_{n}={ }_{2}^{1}\left(z_{n}+\frac{1}{z_{n}}\right)$. Thus $\lambda_{n}$ is the image of $z_{i j}$ under the map from the interior of the unit disc to the complement of the segment $[-1,1]$, taking $z=0$ into $\lambda=\infty$.

2. Let $F_{\alpha}$ denote the space of Taylor series $\sum a_{n} z^{n}$ with norm $\left\|z^{n}\right\|^{2}=$ $n ! \mid \Gamma(\alpha+n+1)$, where $-1<\alpha<\infty$. Clearly $F_{\alpha}$ has the same sets of uniqueness as $D_{-\alpha}$ for $-1<\alpha \leqq 0 . F_{1}$ is the Bergmann space $A_{2}$ of Section IV. We establish an isometry between $F_{\alpha}$ and $L_{2}(0, \infty)$ by the correspondence

$$
z^{n}\left(\frac{\Gamma(\alpha+n+1)}{n !}\right)^{b} \leftrightarrow t^{\alpha / 2} e^{-t / 2}\left(\frac{n !}{\Gamma(\alpha+n+1)}\right)^{3} L_{n}^{(\alpha)}(t),
$$

where $L_{n}^{(\alpha)}(t)$ are Laguerre polynomials. The reproducing kernel in $F_{\alpha}$ corresponds to

$$
t^{\alpha / 2} e^{-t / 2} \sum_{0}^{\infty} \bar{\zeta}^{n} L_{n}^{(\alpha)}(t)=(1-\bar{\zeta})^{-\alpha-1} e^{-t \bar{\zeta} /(1-\bar{\zeta})}
$$

From this we readily deduce: a necessary and sufficient condition that $\left\{z_{n}\right\}$ be a set of uniqueness in $F_{\alpha}$ is that the functions $\left\{t^{\alpha / 2} e^{-\lambda_{n} t}\right\}$ be complete in $L_{2}(0, \infty)$. Here $\lambda_{n}=\left(1+z_{n}\right) /\left(1-z_{n}\right)$ is the image of $z_{n}$ under a conformal map of $|z|<1$ onto $\operatorname{Re} \lambda>0$. For $\alpha=0$ this is Müntz's theorem ([1], Chapter 1), i.e., convergence of the (half-plane) Blaschke product formed from the $\lambda_{z z}$ is the necessary and sufficient condition for completeness of $\left\{e^{-\lambda_{n} t}\right\}$ in $L_{2}(0, \infty)$. 
From Theorem 4 we know the exact characterization of the real positive zeros of functions of class $F_{\alpha}$ (namely, $\left.\sum\left(1-z_{n}\right)<\infty\right)$. Translating this to $L_{2}(0, \infty)$ we have: if $\lambda_{n}$ are real and positive and if $p>-\frac{1}{2}$, then $\left\{t^{p} e^{-\lambda_{n} t}\right\}$ are complete in $L_{2}(0, \infty)$ if and only if $\sum \lambda_{n}^{-1}=\infty$. Because Theorem 4 goes far beyond the spaces $F_{\alpha}$, this last result is undoubtedly true with $t^{p}$ replaced by a more general class of functions. It would be of interest to identify this class. We remark that Theorem 5 leads to a sufficient condition for completeness of $\left\{t^{p} e^{-\lambda_{n} t}\right\}$ in the more general case $\operatorname{Re} \lambda_{n}>0$.

3. The method of the preceding paragraph must be modified if we wish to obtain an analogous "closure" formulation of the uniqueness problem for the class $D$ (the case $\alpha=-1$ ). Working with the equivalently normed space $D^{\prime \prime}:\left\|z^{n}\right\|^{2}=n+1(n=0,1, \ldots)$ we set up an isometry with $L_{2}(0, \infty)$ by

$$
\frac{z^{n}}{\sqrt{n+1}} \leftrightarrow \frac{t^{\frac{1}{5}} e^{-t / 2} L_{n}^{(1)}(t)}{\sqrt{n+1}} .
$$

The image of the kernel function is

$$
t^{\frac{1}{2}} e^{-t / 2} \sum_{0}^{\infty} \frac{L_{n}^{(1)}(t) \bar{\zeta}^{n}}{n+1}=\frac{1-e^{-t^{\overline{5}} /(1-\bar{\zeta})}}{t}
$$

This formula may be obtained by integrating the following identity with respect to $w$ :

$$
\sum_{0}^{\infty} L_{n}^{(1)}(t) w^{n}=e^{-t w /(1-w)}(1-w)^{-2}
$$

From (22) we have: the sequence $\left\{z_{n}\right\}$ is a set of uniqueness for the class $D$ if and only if the functions $\left\{\left(e^{-t}-e^{-\lambda_{n} t}\right) / \sqrt{t}\right\}$ are complete in $L_{2}(0, \infty)$, where $\lambda_{n}=\left(1+z_{n}\right) /\left(1-z_{n}\right)$.

4. As a final illustration we consider the Hilbert space $H$ of entire functions $\sum a_{n} z^{n}$, where $\left\|z^{n}\right\|^{2}=n !$. This space, whose reproducing kernel is $e^{\bar{\zeta} z}$, has many interesting properties. For example, the (unbounded) operator of multiplication by $z$ is adjoint to the operator of differentiation. This space was studied recently by V. BarGmann [Comm. Pure Applied Math. 14 (1961)] with an eye to quantum-theoretical applications. The sets of uniqueness for $H$ are not known. Here we only point out their connection with another. interesting closure problem on $L_{2}(-\infty, \infty)$ (this result is implicit in Bargmann's paper). Under the isometry

$$
\frac{z^{n}}{\sqrt{n !}} \leftrightarrow \frac{(2 \pi)^{-1} e^{-t^{2} / 4} H_{n}(t)}{\sqrt{n !}},
$$

where $H_{n}(t)$ is the $n^{\text {th }}$ Hermite polynomial, the image of the kernel function is

$$
(2 \pi)^{-1} e^{-t^{2} / 4} \sum_{0}^{\infty} \frac{H_{n}(t)}{n !} \bar{\zeta}^{n}=e^{t \bar{\zeta}-\bar{\zeta}^{2} / 2} .
$$


Hence: a sequence $\left\{z_{n}\right\}$ is a set of uniqueness for $H$ if and only if the functions $\left\{e^{-t^{2} / 4} e^{z_{n} t}\right\}$ are complete in $L_{2}(-\infty, \infty)$.

The results of this section indicate that it would be of some interest to make a general study of completeness of exponentials in weighted $L_{2}$ spaces.

\section{Bibliography}

[1] Achiezer, N. I.: Theory of approximation. New York 1956. (Russian edition: Moscow 1947.)

[2] ARonszajN, N.: La théorie générale đes noyaux reproduisants et ses applications. Proc. Cambridge Philos. Soc. 39, 133-153 (1943).

[3] - Theory of reproducing kernels. Trans. Amer. Math. Soc. 68, 337-404 (1950).

[4] CARleson, L.: On a class of meromorphic functions and its associated exceptional sets. Uppsala 1950 (Chapter 2, Theorem 2.2).

[5] - On the zeros of functions with bounded Dirichlet integrals. Math. Z. 56, 289-295 (1952).

[6] - A representation formula for the Dirichlet integral. Math. Z. 73, 190-196 (1960).

[7] Hardy, G. H.: Divergent Series. Oxford 1949.

[8] Hayman, W.: On Nevanlinna's second theorem and extensions. Rend. Circ. Mat. Palermo 2, 346-392 (1953).

[9] LokkI, O.: Utber analytische Funktionen, deren Dirichletintegral endlich ist, und die in gegebenen Punkten vorgeschriebene Werte annehmen. Ann. Acad. Sci. Fennicae 39, 1-57 (1947) (especially pp. 47-49).

[10] Oppenherm, A.: Inequalities connected with definite Hermitian forms. J. London Math. Soc. 5, 114-119 (1930).

[11] PRivaloff, I. I.: Randeigenschaften analytischer Funktionen. Berlin 1956.

[12] Schur, I.: Uber einige Ungleichungen im Matrizenkalkïl. Prace Mat.-Fiz. 44, $353-370(\$ 3)(1937)$.

[13] Wiтtich, H.: Neuere Untersuchungen über eindeutige analytische Funktionen. Berlin 1955 (Chapter 10).

New York University, New York $3, N . Y$.

University of Michigan, Ann Arbor, Mich.

(Received August 10, 1962) 